\title{
CHALLENGES IN TARGETING AREAS FOR PUBLIC ACTION. TARGET AREAS AT THE RIGHT PLACE AND AT THE RIGHT TIME
}

Julie Vallée

CNRS, UMR Géographie-cités

13 rue du Four, 75006 Paris, France

Correspondence to: julie.vallee@ parisgeo.cnrs.fr

While some public interventions are implemented across a whole country and concern everyone, many public interventions focus only on a segment of the population (priority groups) or a part of the territory (priority areas). Targeting groups (i.e. children, elderly people, pregnant women, smokers, migrants, etc.) has long been a strategy to reduce social and health issues. Recently, targeting areas has emerged as an attractive way to implement public action. However, area-based interventions raise significant challenges for policymakers, especially when they want to kill two birds with one stone by combining two targets: priority groups (i.e. for whom health issues are frequent) and priority areas (i.e. for whose attributes impacting health outcomes gain to be modified).

\section{Area-based initiatives: what make them so appealing?}

In many countries, policies targeting a limited number of specific areas have gained in importance. These area-based initiatives result from two rationales. Some areas are targeted because they are places where there is a concentration of people affected by health problems (cf. spatial segregation). Other areas are targeted because the area attributes themselves are involved in the production of health problems (cf. neighborhood effects). Beyond these two not necessarily convergent rationales, at least five driving factors explain why policy-makers find area-based initiatives appealing [1-3]: (i) Area attributes appear to be more easily modifiable and controllable than individual attributes; (ii) Implementing initiatives in a limited number of areas seems to be cost effective notably when issues are spatially concentrated and cumulative; (iii) Functioning as a politically correct euphemism, it conveniently avoids the formulation of issues explicitly linked with 'minority groups'; (iv) Ongoing decentralization processes (meeting citizen expectations for enhanced local democracy and community empowerment) leave local governance structures with the task of designing initiatives in the areas where needs are found to be the greatest; (v) With the increasing availability of precisely localized data, local level diagnosis have become systematic and have encouraged policy-makers to adapt their actions to local specificities. For all these reasons, area-based interventions become strategic in public health actions. However, policy-makers still face some challenges to implement their local interventions at the right place and at the right time (Fig. 1). 


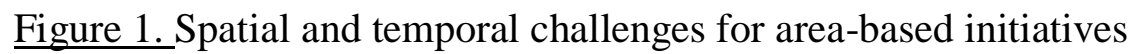

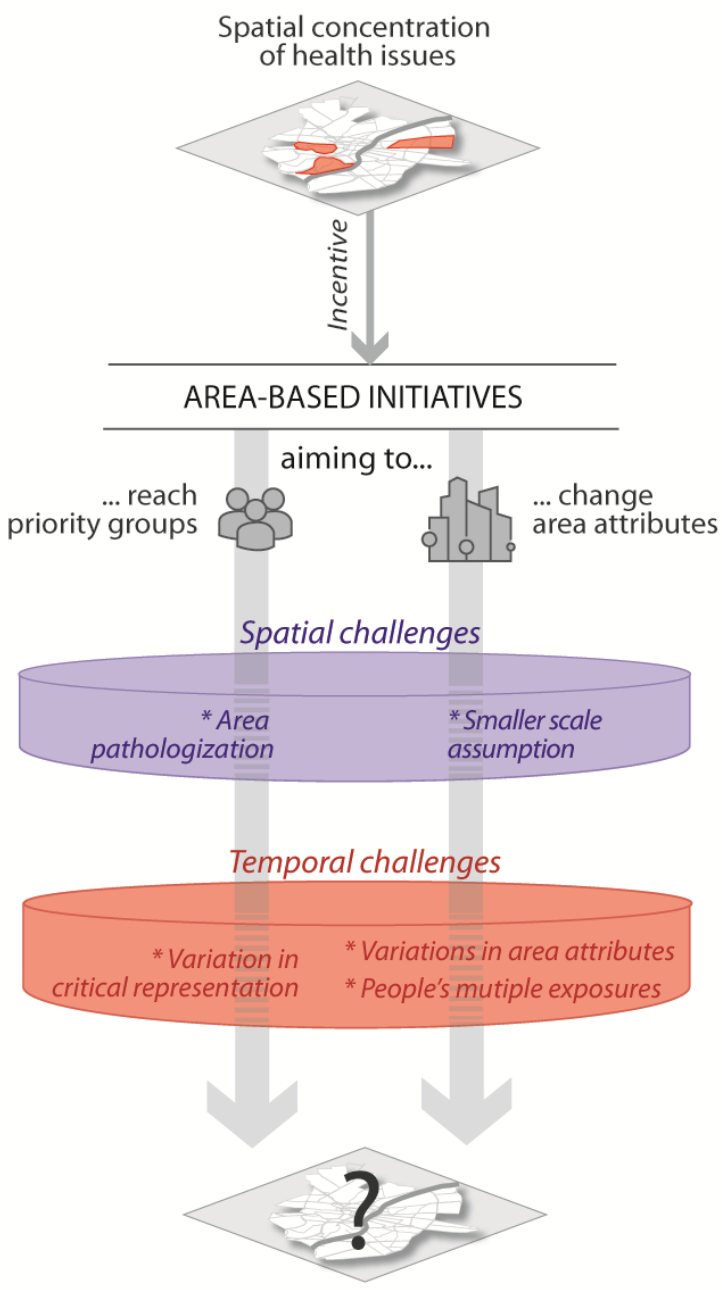

\section{Target areas across the scales}

When adopting an area-based approach in order to reach priority groups, policy-makers may translate societal problems into problems that concern only small, circumscribed areas or 'poverty traps'. Actually, the underlying causes of health issues being concentrated in some specific areas are primarily of a structural nature and are, therefore, external to the local communities where their effects are most acute [4]. Placing emphasis on areas to solve people's health issues may lead to fall into reverse ecological fallacy and see areas as kingsize individuals - to paraphrase Hosfstede speaking about cultures [5]. Such *area pathologization* (Fig. 1) - sometimes called 'spatialism' or 'localism' - is indicative of neoliberal urban policies which muddy the waters by confounding problems in the city with problems of the city [6]. Moreover, these policies may in return contribute to strengthening the discrimination and stigmatization of residents on the basis of their neighborhood of residence.

With the recent popularity of local level diagnoses that are often based on population-based attributes comes also another scale challenge in defining the target areas for public action. When using population-based attributes as a proxy for individual attributes, it is now frequent 
Vallée J, 2017. Challenges in targeting areas for public action. Target areas at the right place and at the right time. Journal of Epidemiology and Community Health..Vol 71 No 10, 945-946. \{10.1136/jech-2017-209197\}.

to select areas as small as possible to avoid falling into the well-known ecological fallacy [7]. While this common-sense practice (to be closest to the people targeted) is appropriate for area-based initiatives aiming to reach priority groups, it is much more debatable for initiatives aiming to change area attributes involved in the production of health issues (cf. neighborhood effects). In this case, it is perilous to consider the *smallest scale as the best* (Fig. 1). It can lead to increase the spatial lag between the underlying causes of a concentration of health issues and the action scales chosen to reduce them [8].

\section{Target areas around the clock}

Besides spatial scales, time scales also matter when targeting areas for public action. A first temporal challenge arises when policy-makers define the nature of areas (and their priority level) from the concentration of people in priority groups residing in these areas. By proceeding in this manner (often from local level diagnosis based on census-based data), one assume that people do not move outside their residential area during the day. When interventions (such as health promotion campaigns) intended for priority populations are implemented during the day in areas where people with health issues are highly concentrated during the night, it may lead to ineffective results, notably if these daytime initiatives target some areas where priority groups have gone off and if, inversely, they do not target the areas where the priority groups have gone to spend the day. The critical representation (i.e. the effectiveness of area-based targeting in reaching people in priority groups) has rarely been empirically tested [2], and only from residential-based estimates [9]. It would therefore be interesting to investigate *variation in critical representation* (Fig. 1), i.e. how representation of priority groups within priority areas change (or not) around the clock. Such an approach, inspired from time-geography, may help to link the temporality of public health interventions with people's daily mobility and to improve the ability of an intervention to reach the people they target (see Fig. 2A for an illustrated example).

Two other temporal challenges occur when policy-makers define the nature of areas (and their priority level) from their attributes at a given point in time. By proceeding in this manner, the first danger is to assume that there are not *variations in area attributes* over 24 hours (Fig. 1), without for example, considering change in air pollution, opening and closing times of facilities or changes in the neighborhood social composition throughout the day [10]. The second danger is to assume that areas with negatives attributes should necessarily be considered as critical, whatever people's daily mobility and *multiple exposures* (Fig. 1). Excluding places where people have regular daily activities can lead to a 'local trap' [11] since neighborhood effects go beyond neighborhoods of residence alone. To take an example in the Paris region, it has been shown that low medical density increases the risk of delayed health screening only for residents whose activity space is limited to their neighborhood of residence [12]. Areas with negative attributes should not to be considered to be of the same priority level regardless of whether the people visiting these areas (i) remain there all the day long or only move to areas with the same attributes, or (ii) move to and spend much of the day in areas with positive attributes. Actually, it would be beneficial that target areas result from the combined location of negative areas attributes and people with high exposure over 24 hours (see Fig. 2.B for an illustrated example). It would prevent policy-makers from going 
Vallée J, 2017. Challenges in targeting areas for public action. Target areas at the right place and at the right time. Journal of Epidemiology and Community Health.. Vol 71 No 10, 945-946. \{10.1136/jech-2017-209197\}.

after the wrong target when they plan interventions to reduce health inequities. Agent-based modeling or geovizualisation tools (for example the Mobiliscope offering an interactive exploration of people's mobility within metropolitan areas over the 24 hours a day [13]) may be helpful in the investigation of spatio-temporal dynamics.

Figure 2. Target areas around the clock

A. Target areas for interventions aiming to reach people (e.g. prevention campaign intended for smokers)
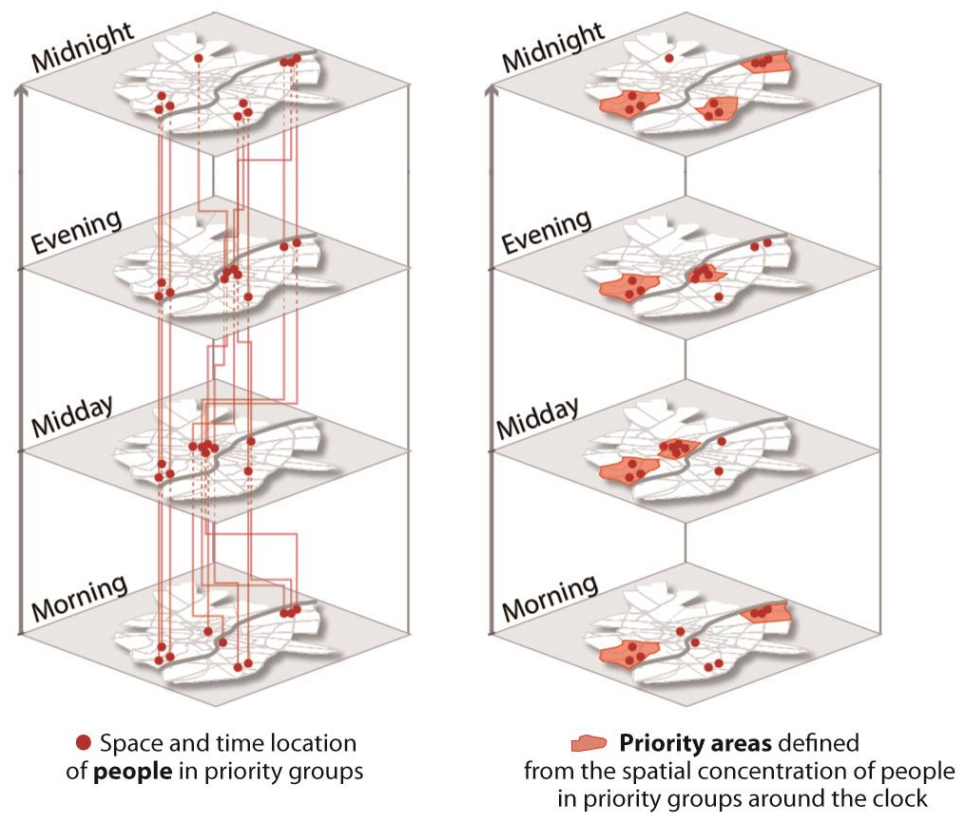

B. Target areas for interventions aiming to change area attributes (e.g. such as campaigns to improve air quality)
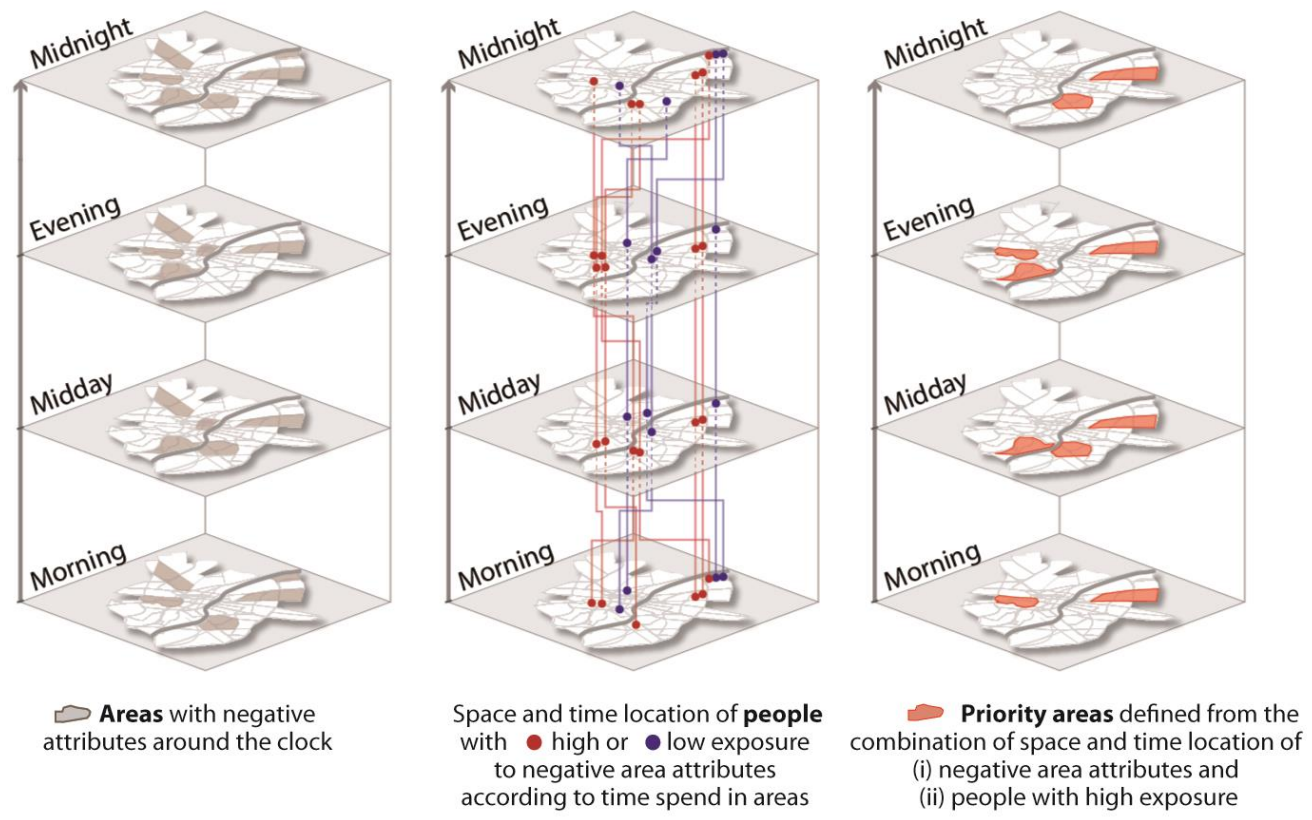
A temporal approach remains a blind spot in the definition of target areas for public actions. While the decision to target areas results from two different rationales (either to reach priority people or to change the area-level attributes themselves), there is a crucial shared need to account for people's daily mobility when one is aiming to reach all people in need of support (instead of just those people who are residing in a concentration area) or to change negative area-attributes to which populations are effectively exposed. If it is true that initiatives related to housing conditions or initiatives implemented during the night may be efficiently defined from a residential-based approach, as is traditionally done, the same cannot be said for initiatives aiming to change the environment to which people are exposed daily or for initiatives implemented during the day.

To conclude, we encourage researchers and public actors to keep in mind that area-based initiates would greatly benefit from seeing areas as dynamic systems over different spatial and time scales. Additional work and discussions are needed to quantify how much moving away from the traditional approach based both on areas of residence and resident population improve efficiency of area-based initiatives.

\section{Acknowledgements}

Thanks to Constance Lecomte for her support in putting together the figures, to Clémentine Cottineau for insights on an earlier version of this paper, to Guillaume Le Roux and Hadrien Commenges for exciting discussions about social segregation around the clock and to every member of the Mobiliscope (http://mobiliscope.parisgeo.cnrs.fr/) and RelatHealth (http://relathealth.parisgeo.cnrs.fr/) projects.

\section{Funding Statement}

The RelatHealth project and the Mobiliscope project received support from the French Government, through the Agence Nationale de la Recherche within Idex Universite Sorbonne Paris Cités (ANR-11-IDEX-00005-02) and Labex DynamiTe (ANR-11-LABX-0046), respectively.

\section{References}

1 van Gent WPC, Musterd S, Ostendorf W. Disentangling neighbourhood problems: area-based interventions in Western European cities. Urban Research \& Practice 2009;2:53-67.

2 Sharpe EK. Targeted neighbourhood social policy: a critical analysis. Journal of Policy Research in Tourism, Leisure and Events 2013;5:158-71.

3 Hancock C, Lelévrier C, Ripoll F, et al. Discriminations territoriales. Entre interpellation politique et sentiment d'injustice des habitants. Marne-la-Vallée, France 2016. 
$4 \quad$ Fletcher DR. A culture of worklessness? Historical insights from the Manor and Park area of Sheffield. Policy and Politics 2007;35:65-85.

$5 \quad$ Hofstede G. Culture's Consequences: Comparing Values, Behaviors, Institutions and Organizations Across Nations. Thousand Oaks CA: Sage Publications 2001.

6 Garnier J-P. Essais sur la ville, la petite-bourgeoisie intellectuelle et l'effacement des classes populaires. Marseille: Agone 2010.

7 Bryere J, Pornet C, Copin N, et al. Assessment of the ecological bias of seven aggregate social deprivation indices. BMC Public Health 2017;17:86.

8 Vallée J, Shareck M. A response to "Examination of How Neighborhood Definition Influences Measurements of Youths' Access to Tobacco Retailers: A Methodological Note on Spatial Misclassification". American Journal of Epidemiology 2014;179:6602.

9 Tunstall R, Lupton R. Is targeting deprived areas an effective means to reach poor people? In: Centre for Analysis of Social Exclusion, ed. CASEpaper. London, UK: London School of Economics and Political Science 2003.

10 Le Roux G, Vallée J, Commenges H. Social segregation around the clock in the Paris region. Journal of Transport Geography 2017;59:134-45.

11 Cummins S. Commentary: investigating neighbourhood effects on health-avoiding the 'local trap'. International Journal of Epidemiology 2007;36:355-7.

12 Vallée J, Cadot E, Grillo F, et al. The combined effects of activity space and neighbourhood of residence on participation in preventive health-care activities: The case of cervical screening in the Paris metropolitan area (France). Health \& Place 2010;16:838-52.

13 Mobiliscope : http://mobiliscope.parisgeo.cnrs.fr/ 